\title{
NEW FORMING SCHEME OF REFERENCE OF LL BAND FOR IN-BAND VIDEO CODING WITH SPATIAL SCALABILITY
}

\author{
Yinji Piao, HyunWook Park \\ Department of Electrical Engineering, Korea Advanced Institute of Science and Technology \\ 373-1 Guseong-dong, Yuseong-gu, Daejeon, Korea
}

\begin{abstract}
In this paper, we proposed a new scheme of forming quarterpixel reference of LL band for in-band video coding with spatial scalability. The recently proposed leaky motion compensation and mode-based temporal filtering methods [5] have reduced the drifting error dramatically. However there still remains drifting error because of the use of high quality reference and the difference between high quality and low quality references [5]. In our method, a new reference is formed which gives PSNR higher than the low quality reference. The new reference is used instead of low quality reference in leaky and mode-based methods and experimental results show that improvement can be achieved in PSNR comparison with the existed ones.
\end{abstract}

Index terms- Wavelet transform, video coding, frequency estimation

\section{INTRODUCTION}

The wavelet transform achieves great success in still image compression such as JPEG2000. Also in video coding, the inherent property of multiscale representation makes wavelet transform become a base of scalable video coding not only in space but also in time domain. Differently from other coders, wavelet-based coders need to encode the bitstream only once but flexibly adapt to heterogeneous terminals and networks, which means quality, resolution and temporal scalabilities are converged in one bitstream. This facility increases the necessity of wavelet-based video coders.

Until now, there are two main 3D wavelet-based video coders. One is inter-frame wavelet coder [1], and the other is in-band wavelet coder [2][3]. Inter-frame wavelet coders perform wavelet transform along temporal axis to generate temporal high pass and low pass frames in time domain. Then spatial wavelet transform is applied to the high pass and low pass frames of temporal filtering. So inter-frame wavelet scheme is also called $\mathrm{T}+2 \mathrm{D}$ motion-compensated temporal filtering(MCTF). In contrast, in-band wavelet coders [2][3] perform 2D spatial wavelet transform for each frame followed by temporal filtering to the spatial subbands. Considering the process order, in-band wavelet scheme is also called 2D+T MCTF. Recently 2D+T+2D MCTF coder [6] has been developed which is similar to the in-band wavelet coders except applying $2 \mathrm{D}$ wavelet transform to the residual of $2 \mathrm{D}+\mathrm{T}$ schemes. Although it includes $\mathrm{T}+2 \mathrm{D}$ process, we can treat $2 \mathrm{D}+\mathrm{T}$ and $2 \mathrm{D}+\mathrm{T}+2 \mathrm{D}$ coders as the same type of coders since motion estimation which is the most important part of video coding is performed in wavelet domain.

The most widely discussed topic of in-band wavelet coders is the motion estimation in wavelet domain. Because of the shift-variant property of wavelet transform, wavelet domain motion estimation is ineffective until the low-band shift (LBS) method proposed by Park and Kim [4] solved the shift variant problem and made it possible and effective to perform motion estimation in wavelet domain with shiftinvariance. In general in-band wavelet coders, the motion estimation are performed on the reference of over-complete form of wavelet transform (ODWT) [3].

However, in in-band video coding with spatial scalability, the temporal filtering in the low resolution uses high band signal which is high resolution information to generate overcomplete form of reference frame for motion compensation. This results in serious drifting problem since high band signal is unavailable when decoding at low resolution. Leaky motion compensation and mode-based temporal filtering methods [5] were proposed to reduce drifting error and have been applied to Vidwav evaluation software [6]. Although the two methods dramatically improved the coding efficiency by reducing drifting error, there still exists drifting problem because of the unmatched use of high quality reference at the encoder and decoder. In this paper, we propose a new forming scheme of quarter-pixel reference of LL band and use it instead of low quality reference in leaky motion compensation and mode-based temporal filtering methods. Our purpose is to make a reference which is similar to high quality reference but better than low quality 
reference to reduce the gap of high quality reference and low quality reference.

This paper is structured as follows. In section 2.1 the high quality and low quality references of leaky and mode-based techniques are briefly reviewed. The proposed reference forming scheme is presented in section 2.2. The conducted experiments and their results are given in section 3. Finally, the conclusions on the results and our further works are provided in section 4 .

\section{PROPOSED METHOD}

We first review the definition of high quality reference and low quality references used in leaky motion compensation and mode-based temporal filtering methods in Vidwav evaluation software, and then introduce our proposed method.

\subsection{High Quality and Low Quality References}

In [5], the definition of high quality reference and low quality references was given as follows. The high quality reference is showed in Fig.1. (a), which forms the 1/4-pixel reference of LL band by using LL band and its corresponding high bands to do inverse wavelet transform and over-complete wavelet transform and then perform $2 \times 2$ sinc interpolation. Low quality reference showed in Fig.1.(b) is formed by LL band with direct $4 \times 4$ sinc interpolation.

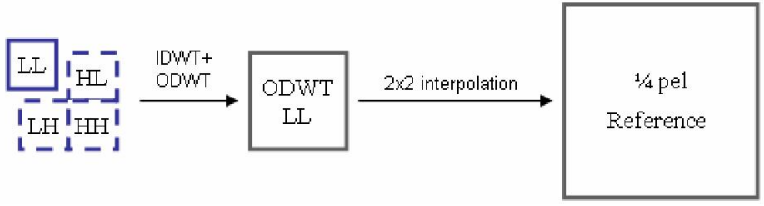

(a) High quality reference

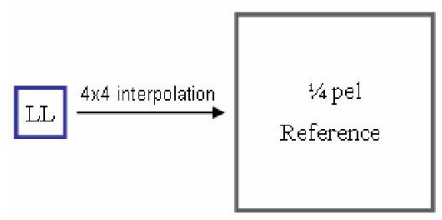

(b) Low quality reference

Fig.1.The high-quality and low-quality references for motion estimation

The leaky motion compensation method in Vidwav evaluation software uses the motion vector searched with high quality reference but compensates with high quality and low quality references by a given weight.

$$
\text { ref }=\text { ref_highquality } \times \alpha+\text { ref_lowquality } \times(1-\alpha)
$$

However, in the decoder the low quality reference uses the motion vector searched with high quality reference which is not the optimal motion vector of low quality reference. This leads to unmatched problem of motion vector and reference.

The mode-based temporal filtering predicts the motion of each macroblock with both high quality and low quality references and calculates the rate distortion cost of each of them. With a given threshold the ratio of rate distortion costs of two modes is measured and the final mode of reference will be determined as follows,

$$
r e f=r e f_{-} \text {highquality, if } \quad \text { ratio }=\frac{\min \left(R D_{-} C_{\text {Cost }}{ }_{\text {low }}\right)}{\min \left(R D_{-} \text {Cost }_{\text {high }}\right)}>\text { threshold }
$$$$
\text { ref }=\text { ref_lowquality, otherwise. }
$$

The mode-based temporal filtering also has unmatched problem in the decoder side because the high quality mode is not available when decoding at low resolution.

\subsection{Proposed Method}

We propose a new 1/4 pixel reference of LL band which can substitute the low quality reference to reduce the gap of high quality and low quality references. It also can be used as high quality reference both in encoder and decoder for the purpose of removing the drifting problem.

When decoding at low resolution, corresponding high bands are not available. So we generate new high bands which may contain some information of original high bands. Because Daubechies 9/7 tap analysis filter bank used in wavelet transform has finite filter taps, the low band signal contains some information of high band signal. We try to make new high bands information with LL band in the following method. The scheme of forming $1 / 4$ pixel LL reference is showed in Fig.2.

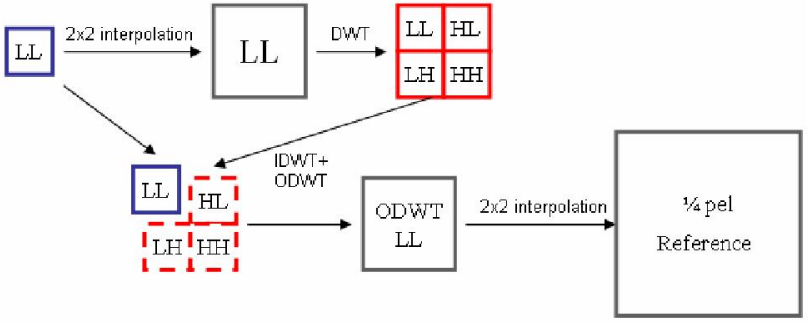

Fig.2. LL band with new high band scheme

First, $2 \times 2$ sinc interpolation is applied to the LL band, and then performs the 2D wavelet transform to get new high bands. Original LL band together with these new high bands follow the inverse wavelet transform and over-complete 

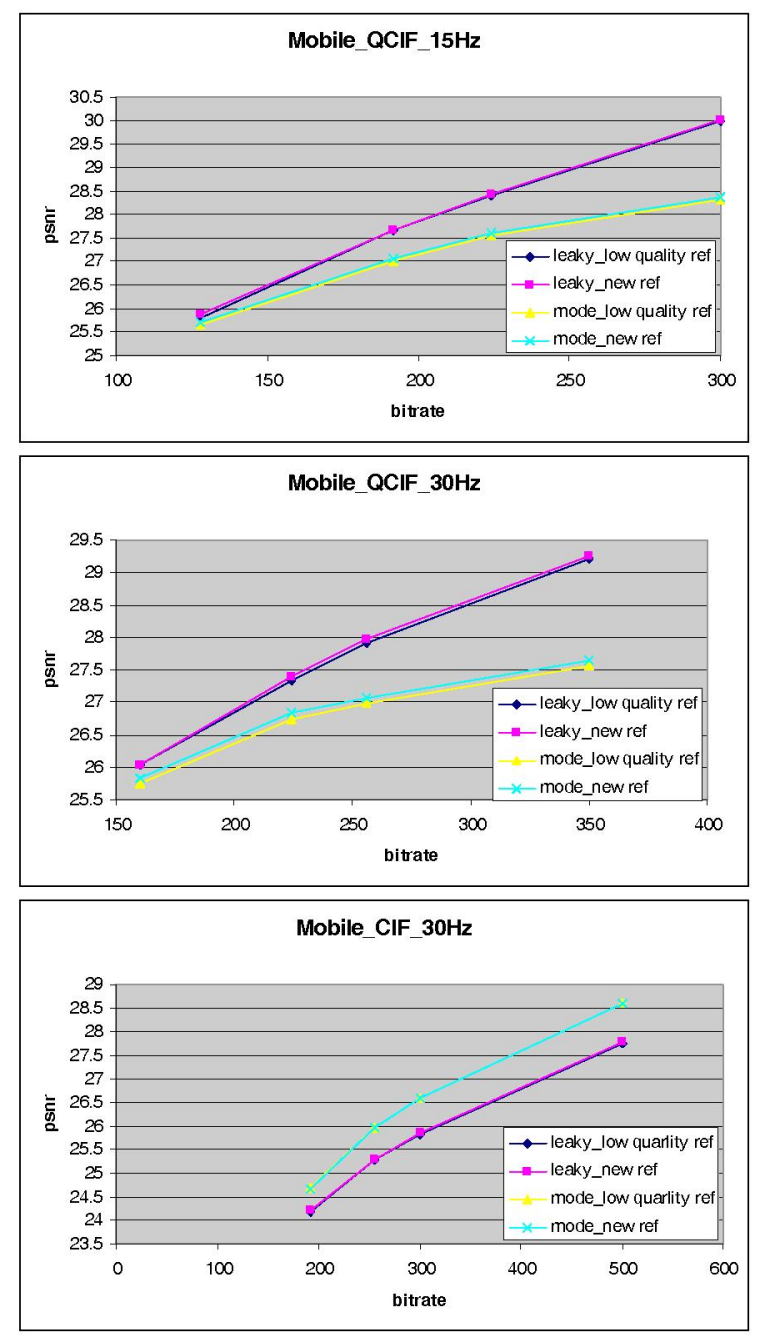

Fig. 3. Performance for Mobile CIF sequence in previous schemes using new reference instead of low quality one

wavelet transform, finally complete with the $2 \times 2$ sinc interpolation.

The proposed scheme is similar to the forming scheme of high quality reference since it also uses the process of IDWT $\rightarrow$ ODWT $\rightarrow 2 \times 2$ interpolation. However the proposed one doesn't use the unavailable high bands but new high bands generated with the existed LL band signal.

\section{EXPERIMENTAL RESULTS}

In this section we will give two sets of experimental results. Both of them are implemented in Vidwav evaluation software. Mobile calendar CIF sequence (100 frames) and foreman CIF sequence (100 frames) are used as test sequences in the experiments.
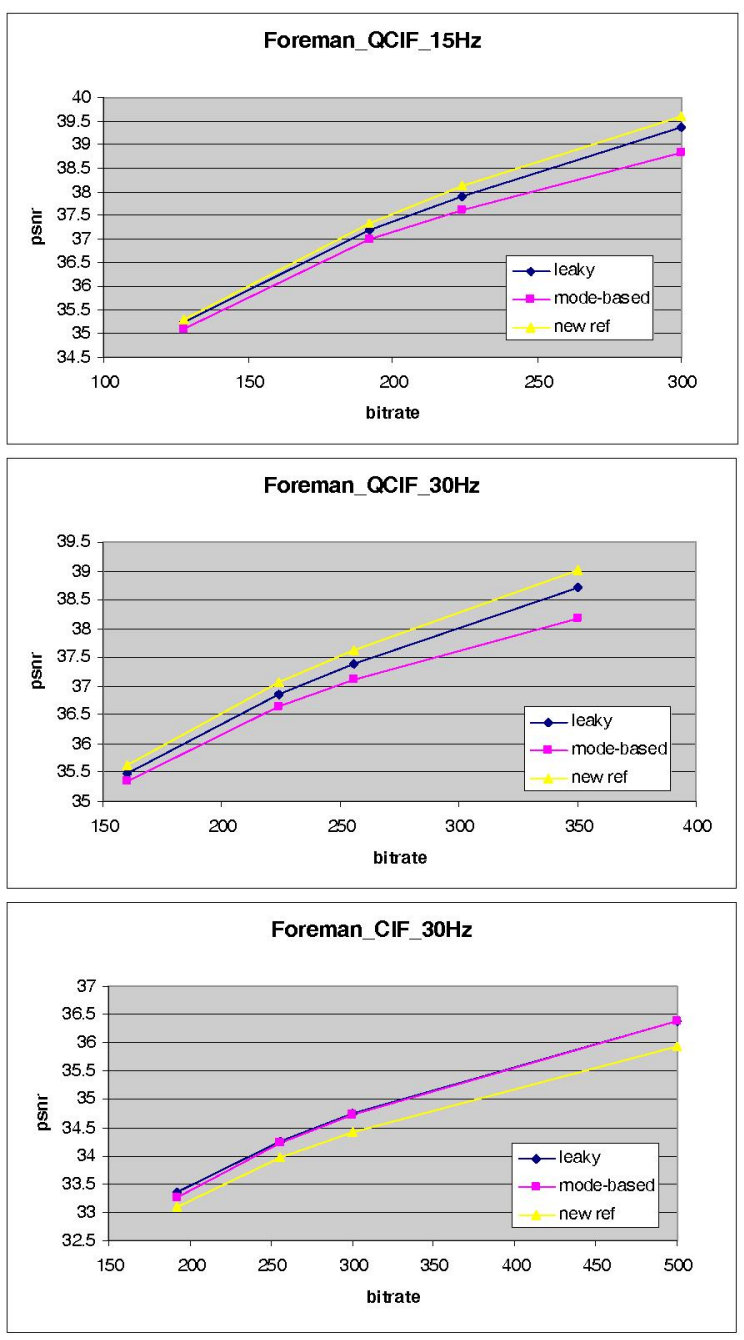

Fig.4. Performance for Foreman CIF sequence with different schemes

In the first experiment, we substitute the low quality reference in the leaky motion compensation and modebased temporal filtering methods with our new reference. The average luminance PSNR results in Fig.3. show that the leaky motion compensation and mode-based temporal filtering with our new reference has higher PSNR than that with the low quality reference. At QCIF resolution with full temporal rate, nearly $0.1 \mathrm{~dB}$ gain can be obtained in modebased temporal filtering. Even at full resolution our method is a little bit higher than leaky and mode-based method with low quality reference.

In the second experiment the new reference is used in motion prediction and compensation both in the encoder and decoder. The purpose of this scheme is to solve the unmatched problem of motion vector and reference. In other words, new reference is used as the high quality reference 
although there is much difference between two of them. The average luminance PSNR comparison with leaky motion compensation and mode-based temporal filtering methods are presented in Fig.4. The results show that when spatial scalability is required the above method gives up to $0.4 \mathrm{~dB}$ improvement compared to the leaky motion compensation method and $0.8 \mathrm{~dB}$ improvement compared to the modebased temporal filtering method. However, at full resolution, it loses nearly $0.5 \mathrm{~dB}$ performance compared to the leaky motion compensation and mode-based temporal filtering methods. Because when decoding at full resolution the high band signal is available and high quality reference can be formed.

\section{CONCLUSION}

In this paper, we introduced a new scheme of forming quarter-pixel LL band reference which was made by LL band with new high bands. The new reference showed better result than low quality reference when applied to the leaky motion compensation and mode-based temporal filtering method. We also used the new reference as high quality reference in both encoder and decoder for the purpose of removing the unmatched problem of motion vector and reference. Experimental results proved that the proposed scheme outperformed the leaky and mode-based method when spatial scalability was required. However, our new reference still has much difference from the high quality reference because of the impossibility to make exact high bands with low band signal. If we can utilize as much high band information as possible in the low band, it is possible to make new reference as close as high quality reference.

\section{REFERENCES}

[1] J.R.Ohm, Mihaela van der Schaar, J.W.Woods, "Interframe Wavelet Coding-Motion Picture Representation for Universal Scalability", EURASIP Signal Processing: Image Communication, Vol.19, pp 877-908, Issue 9, Oct. 2004

[2] Y.Andreopoulos, A.Munteanu, J.Barbarien, M.van der Schaar, J.Cornelis and P.Schelkens, "In-band motion compensated temporal filtering", EIRASIP Signal Processing: Image Communication, Vol. 19, No.7, pp 653-673, Aug. 2004

[3] J.C.Ye, Mihaela van der Schaar, "Fully Scalable 3-D Overcomplete Wavelet Video Coding using Adaptive Motion Compensated Temporal Filtering", Proceedings of VCIP, Lugano, Switzerland, July 2003

[4] H.W. Park, H.S. Kim, "Motion Estimation Using LowBand-Shift Method for Wavelet-Based Movidng-Picture Coding", IEEE Trans. On Image Processing, Vol 9, No.4, pp 577-587, Apr. 2000.
[5] ISO/IEC JTC1/SC29/WG11 M11681 "Improvement for In-band Video Coding with Spatial Scalability", \#71 MPEG Meeting, Hong Kong, Jan. 2005

[6] ISO/IEC JTC1/SC29/WG11 N7334, "Wavelet Codec Reference Document and Software Manual", \#72th MPEG Meeting, Poznan, July 2005 